\title{
TRAJECTORY BASED DROPLET COLLISION MODEL FOR SPRAY MODELING
}

\author{
Ö. O. TAk̦KIRAN ${ }^{\mathrm{a},{ }^{*}}$ and M. ERGENEMAN ${ }^{\mathrm{b}}$ \\ ${ }^{a}$ Domestic Research Division, Inventory Control Center, Golcuk, Kocaeli \\ ${ }^{b}$ Automotive Division, Faculty of Mechanical Engineering, Istanbul Technical University, 34469 Maslak, \\ Istanbul, Turkey
}

\begin{abstract}
This study presents a droplet collision model which takes into account trajectories of colliding droplets. Droplet collision dynamics can be represented more realistically by using a trajectory based model since collisions are calculated by the position and velocity vectors of droplets. Unlike the Ođ̂Rourkeôs model which is used in KIVA code, the presented model is not mesh dependent. The key point of the model is defining the droplet impact parameter. In this study, a new calculation method of impact parameter, which is based on binary droplet collision model, is presented. The impact parameter of two colliding droplet is obtained by following their colliding trajectory. The algorithm was implemented into the KIVA3V Rel2 code and obtained droplet calculations were compared with Ođ̃Rourkeôs collision model. Binary collision of droplet pairs were taken into account and the impact parameter of two colliding droplets was calculated from their position and velocity vectors. The new model represents the physical process of collusion more accurately since it uses colliding droplets in the calculations.
\end{abstract}

Keywords: Droplet collision, impact parameter, spray modeling.

* Corresponding author: Tel: +90 5326013351

E-mail address: taskiranoz@itu.edu.tr

\section{Introduction}

The numerical spray modeling offers very important and valuable information for the calculation of diesel spray combustion. KIVA3V R2 code, which is a Computational Fluid Dynamics (CFD) program used for engine research, describes the spray dynamics and combustion process with related sub-models, such as breakup, collision, evaporation models and etc.

Droplet collision is an important physical part of numerical spray calculations. Droplet collisions affect the spray propagation, droplet evaporation and distribution by changing the droplet number and size. In the standard KIVA3V R2 code which uses Discrete Droplet Model (DDM), the collisions may give two physical results; coalescence and grazing [1]. In fact, there are other important physical results of droplet collisions [2]. Qian and Law [3] defined the results of collision of hydrocarbon droplets as bouncing, coalescence, reflexive separation, stretching separation (grazing) and shattering collision. The standard KIVA code is based on the OâRourkeôs [4] collision sub-model and the calculations proceeds with the basic steps given below. Droplets groups are represented by the parcels and all droplets in a parcel behave in the same way. The collision calculation is performed for the pair of particles if they are in the same cell. The collision probability Pn, that a larger parcel undergoes ñò collisions with smaller parcel, is assumed to follow a Poisson distribution [1].

The standard collision model has the disadvantage of being mesh dependent since there is a requirement that the parcels must be in the same cell to be taken into the collision calculation. A finer mesh would include less droplet parcels and the collision probability depends on cell dimensions. The collision results are important especially for intersecting sprays. Though they present fast solutions for spray calculations, the trajectory of the droplets, which is important for spray dynamics, are ignored in the stochastic methods. It was shown in Nordinôs thesis [5] that standard KIVA model gives mesh depended results. Nordin proposed a trajectory based model for droplet collision, which reduces mesh dependency [5]. Nordinô model includes a new probability distribution function. However, in Nordinôs collision model, impact parameter of colliding droplets is obtained by choosing a random number similar to Ođ̊Rourkeôs one. In the literature, there are also other droplet collision algorithms to reduce mesh dependency [6,7] and there are developed models for describing the outcome of droplets collision outcomes $[8,9]$.

Trajectory based models have to use definite and explicable parameters unlike the stochastic models do. The main purpose of this study is to present a new calculation method for defining the impact parameter of droplet collision. This new method eliminates choosing random number for impact parameter calculation and enables to calculate it from droplet velocity and position vectors data. The trajectory based collision model was implemented into the KIVA3V Rel2 code and results of droplet calculations were compared with collision model of the Ođ̃Rourke which was accepted as the standard model [6]. In the last section of this study, by considering 
macroscopic characteristics, the KIVA spray simulation results have been compared with experimental spray images that were obtained by injecting fuel into a constant volume vessel [10].

\section{Theoretical background}

Standard KIVA collision model uses a probability distribution function for parcels collisions [1], which was based on Ođ̂Rourke collision model [4]. The collision probability Pn, that gives the probability of a larger parcel undergoes $\mathrm{n}$ collisions with smaller parcel is proposed to follow a Poisson distribution.

$$
P n=e^{-\bar{n}} \frac{\bar{n}^{n}}{n !}
$$

with mean value $\bar{n}=v \Delta t$, where $q 0$ is the computational time step and the 3 is the collision frequency of the larger parcel. The calculation of the collision frequency, 3, is important since it depends on the cell volume as parameter. It is defined as;

$$
v=\frac{N_{2}}{\delta V} \pi\left(r_{1}+r_{2}\right)^{2}\left|\mathbf{u}_{1}-\mathbf{u}_{2}\right|
$$

where $r$ is radius of the parcel, the subscripts 1 and 2 represents the larger and smaller parcels, N2 is the number of droplets in the smaller parcel and ŭV is the volume of the cell $[1,4]$.

The collision impact parameter is defined as;

$b=\sqrt{R R}\left(r_{1}+r_{2}\right)$

by using a chosen random number (RR). The impact parameter is important since it helps to determine the outcome of collision. If the collision impact parameter is less than a critical collision impact parameter, $b<b_{c r}$, coalescence occurs after the collision otherwise it is a grazing collision [1]. The value of $b_{c r}$ is calculated from the coalescence efficiency of which details were described in Ođ̂Rourke thesis [4].

Nordin put forward a new trajectory based probability distribution for droplet collisions in order to eliminate the requirement of being the parcels in the same cell [5]. In OôRourkeôs model the parcels have equal chance for collisions whether they are moving towards or away from each other. This is expressed as another weakness of OâRourkeôs formulation. The collision model suggested by Nordin states; ñCollisions between two parcels occurs if their trajectories intersect and if the intersection point is reached at the same time, within the integration stepò [5].

$\mathbf{x}_{1}$

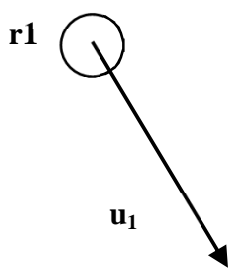

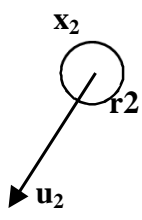

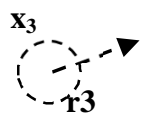

$\mathbf{u}_{3}$

Figure 1 : Collision geometry of Nordin

Nordin [5] suggests two prerequisites to reduce the computational work to be done all parcels. First one is that the parcels in consideration must travel towards each other for a collision as in Fig.1;

$U_{12}=\left(\mathbf{U}_{1}-\mathbf{U}_{2}\right) \cdot \frac{\mathbf{x}_{2}-\mathbf{x}_{1}}{\left|\mathbf{x}_{2}-\mathbf{x}_{1}\right|}$

The second one is the parcels relative displacement must be larger than the distance between them. For example, the parcel number 3, shown with dotted line in Fig. 1, will not be taken into droplet collision calculation.

$U_{12} \Delta t>\left|\mathbf{x}_{2}-\mathbf{x}_{1}\right|-\left(r_{1}+r_{2}\right)$

When the parcels met these requirements, collision occurs if a chosen random number $\xi \in[0,1]$, is less than the probability P. Nordin [5] propose new collision probability P, as;

$P=\min \left(C_{1} \frac{r_{1}+r_{2}}{\left|\mathbf{p}_{1}-\mathbf{p}_{2}\right|}, 1\right) e^{-C_{2}\left|\alpha_{0}-\beta_{0}\right| / \Delta t}$ 
where $\left|\mathbf{p}_{\mathbf{1}}-\mathbf{p}_{2}\right|$ is the minimum distance between the two trajectories and $C_{1}, C_{2}$ are model constants. The trajectory vectors are defined as;

$\mathbf{p}_{1}=\mathbf{x}_{1}+\alpha \mathbf{U}_{1}, \mathbf{p}_{2}=\mathbf{x}_{2}+\beta \mathbf{U}_{2}$

and if $\alpha_{0} \in[0, \Delta t], \beta_{0} \in[0, \Delta t]$, the solution comes from the requirement below.

$\frac{\partial\left|\mathbf{p}_{1}-\mathbf{p}_{2}\right|}{\partial \alpha, \beta}\left(\alpha_{0}, \beta_{0}\right)=0$

$\breve{U}_{b}$ and $\breve{b}_{0}$ contain the information on collision time. The constant $C_{1}$ is defined as spatial probability decay. If $C_{1}$ is lover than unity, the probability equation (6) allows the droplets, which are otherwise not going to meet each other, to collide. In this case the collision number in the spray may increase.

\section{New calculation model}

In the Lagrangian phase of KIVA algorithm, droplets are traced by their position and velocity data. The stochastic methods introduce probability functions in order to define phenomena under investigation. Though they present fast solutions for spray calculations, the trajectory of the droplets, which is important for spray dynamics, are ignored in the stochastic methods. The new model uses the parcel velocity and position data and calculates the time of droplets collision. The advantage of the new model is deriving impact parameter of the colliding droplets. Figure 2 presents the schematic of calculation method of the droplet collision. The bigger droplet has radius $r_{1}$ and position $x_{1}$.

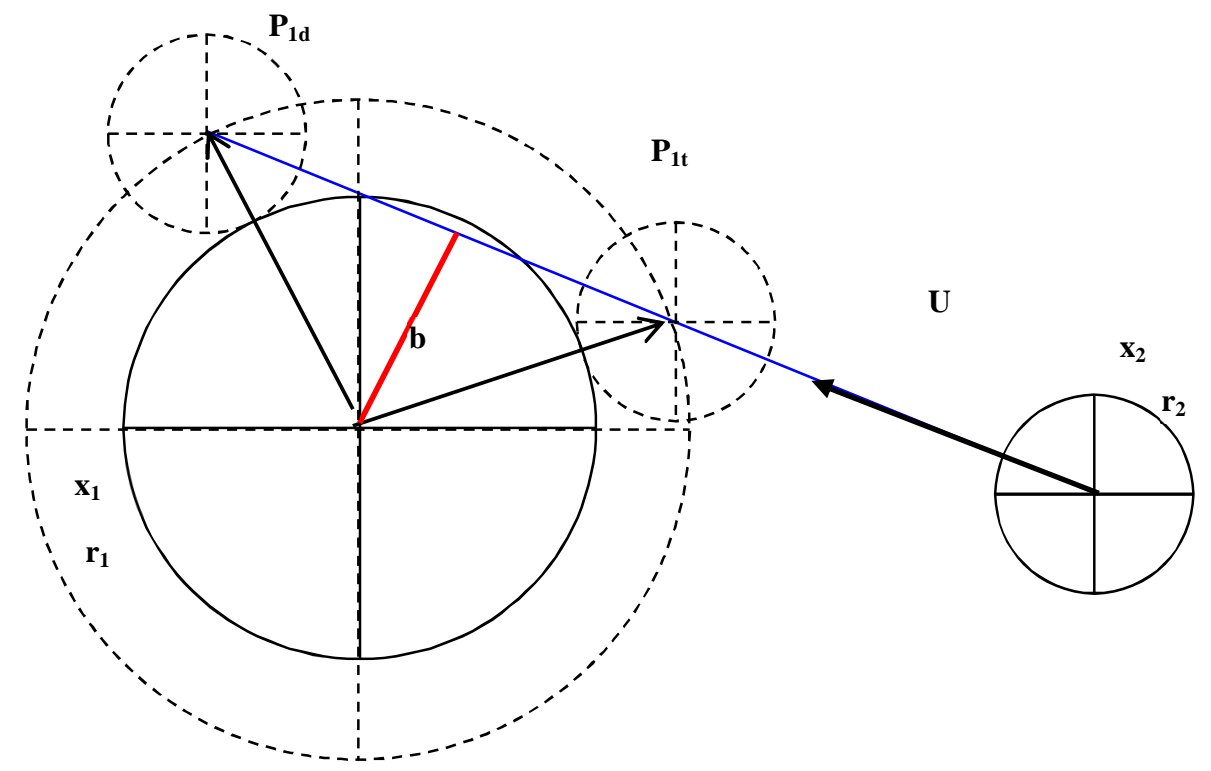

Figure 2 : The representation of new mathematical modeling

At the instant of collision, $\mathrm{t}_{\mathrm{c}}$, the distance between the parcels is;

$\left|\mathbf{x}_{2}\left(t_{c}\right)-\mathbf{x}_{1}\left(t_{c}\right)\right|=r_{1}+r_{2}$

where the position vectors are;

$\mathbf{p}_{1}=\mathbf{x}_{1}+t_{c} \mathbf{u}_{1}$ and $\mathbf{p}_{2}=\mathbf{x}_{2}+t_{c} \mathbf{u}_{2}$

A quadratic equation can be obtained from the above model as follows;

$$
\begin{aligned}
& \left|\left(\mathbf{x}_{2}+t_{c} \mathbf{u}_{2}\right)-\left(\mathbf{x}_{1}+t_{c} \mathbf{u}_{1}\right)\right|^{2}=\left(r_{1}+r_{2}\right)^{2} \\
& \left|\mathbf{x}_{1}-\mathbf{x}_{2}\right|^{2}+2\left(\mathbf{u}_{1}-\mathbf{u}_{2}\right)\left(\mathbf{x}_{1}-\mathbf{x}_{2}\right) t_{c}+\left|\mathbf{u}_{1}-\mathbf{u}_{2}\right| t_{c}^{2}=\left(r_{1}+r_{2}\right)^{2}
\end{aligned}
$$

By introducing $\Delta \mathbf{x}=\mathbf{x}_{1}-\mathbf{x}_{2}, \Delta \mathbf{u}=\mathbf{u}_{1}-\mathbf{u}_{2}$ and $\sigma=r_{1}+r_{2}$, the equation above becomes,

$|\Delta \mathbf{x}|^{2}+2(\Delta \mathbf{u} \Delta \mathbf{x}) t_{c}+|\Delta \mathbf{u}|^{2} t_{c}^{2}=\sigma^{2}$

The smaller positive root of the Eq. (11) gives the collision time, $t_{c}$, and the greater positive root gives the departure time. If $t_{c t}$ and $t_{c d}$ are the times of touching and departing of droplets from each other, the position vectors at the instant of touching time $(\mathrm{t})$, and departing time $(\mathrm{d})$, can be written as follows; 
$\mathbf{p}_{1 t}=\mathbf{x}_{1}+t_{c t} \mathbf{u}_{1}$ and $\mathbf{p}_{2 t}=\mathbf{x}_{2}+t_{c t} \mathbf{u}_{2}$

$\mathbf{p}_{1 d}=\mathbf{x}_{1}+t_{c d} \mathbf{u}_{1}$ and $\mathbf{p}_{2 d}=\mathbf{x}_{2}+t_{c d} \mathbf{u}_{2}$

The distance travelled between the $\mathrm{t}$ and $\mathrm{d}$ times could be obtained by $D_{t d}=|\Delta \mathbf{u}|\left(t_{d}-t_{c}\right)$ and the b length, which is the impact parameter, equals to:

$b=\sqrt{\sigma^{2}-\left(D_{t d} / 2\right)^{2}}$

If $\mathrm{b}$ is smaller than $\mathrm{r}_{1}$, the center of the drop2 doesnâ intersect the drop1, Fig 2. The dimensionless impact parameter $\mathrm{B}$ is $\mathrm{B}=\mathrm{b} / \mathrm{u}$. If $\mathrm{B}=0$, it is a frontal collision (the droplet collide center to center) and if $\mathrm{B}=1$, it is a grazing collision (droplets tangentially pass each other). After obtaining the impact parameter $b$, it is compared with critical impact parameter, $b_{\mathrm{cr}}$ to get the outcome of collision.

\section{Computational Results}

The new model was applied to numerical KIVA3V R2 code with Nordinôs prerequisites (Eq. 4 and 5) to reduce the calculation time. The collision model was tested for five conditions as presented in the Table 1. Standard KIVA3V R2 collision modeling results are listed in the last column. The fuel was injected from a single hole-injector nozzle to $3 \mathrm{MPa}$ and $300{ }^{\circ} \mathrm{K}$ constant volume with $0.6 \mathrm{~ms}$. injection duration. Total particle collisions from injection time to $0.3 \mathrm{~ms}$ are also given in Table 1.

Table 1: Collision model test cases

\begin{tabular}{|l|l|l|l|l|l|l|}
\hline & Case 1 & Case 2 & Case 3 & Case 4 & Case 5 & Std.Mdl. \\
\hline Particle Number & 3000 & 3000 & 6000 & 8000 & 10000 & 3000 \\
\hline Injected Mass & $0.6 \mathrm{mg}$ & $0.3 \mathrm{mg}$ & $0.6 \mathrm{mg}$ & $0.6 \mathrm{mg}$ & $0.6 \mathrm{mg}$ & $0.6 \mathrm{mg}$ \\
\hline Total collisions* & 29173 & 41265 & 105580 & 187618 & 338393 & 1538459 \\
\hline Total coalescence* & 3941 & 5789 & 21947 & 51336 & 128495 & 226710 \\
\hline * from start of injection to $0.3 \mathrm{~ms}$ &
\end{tabular}

In Case 1, 3000 parcel is selected as initial condition to represent the whole spray. The total droplet collisions until $0.3 \mathrm{~ms}$ are 29173. The dimensionless impact parameter distribution of these collisions is depicted in Fig.3. Center-center collisions are less than the tangential collisions, since projected area of colliding droplets is lesser for 0.0-0.1 mm than 0.1-0.2 and so on. The expected impact parameter distribution verifies the correctness of new calculation algorithm.
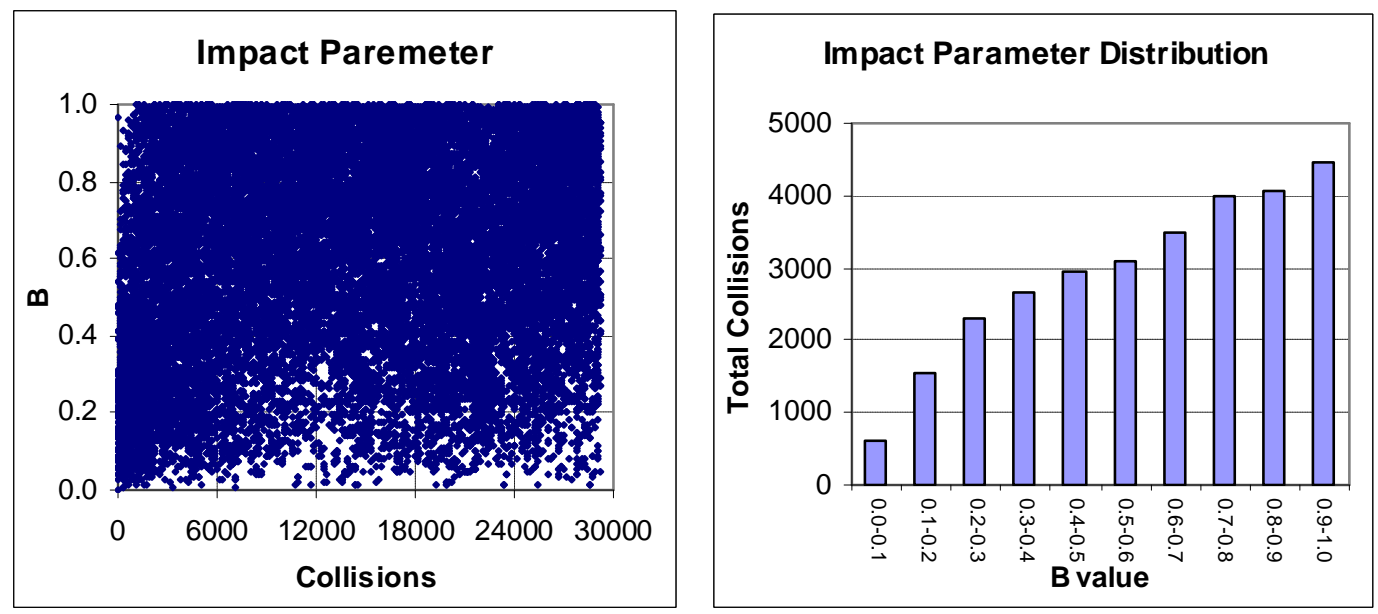

Figure 3 : Impact paremeter distributions, Case 1

Though there are 29173 collisions for Case 1, the coalescence occurs for only 3941 parcels and the other collisions are grazing ones. The reason for different coalescence outcome is related to the high values of critical impact parameter, $\mathrm{B}_{\mathrm{cr}}$. After collision, particles coalescence, if the impact parameter, $\mathrm{B}$, is less than critical impact parameter, $\mathrm{B}_{\mathrm{cr}}$. The critical impact parameter equation, which was used in the study is the same as Ođ̂Rourkeôs ones and it depends on droplet velocity, surface tension and radius [4]. In order to test the effect of velocity, the injected amount is reduced in Case 2. The increase in coalescence was very limited. The coalescence and grazing collision ratios of tested cases are presented in Figure.4. 


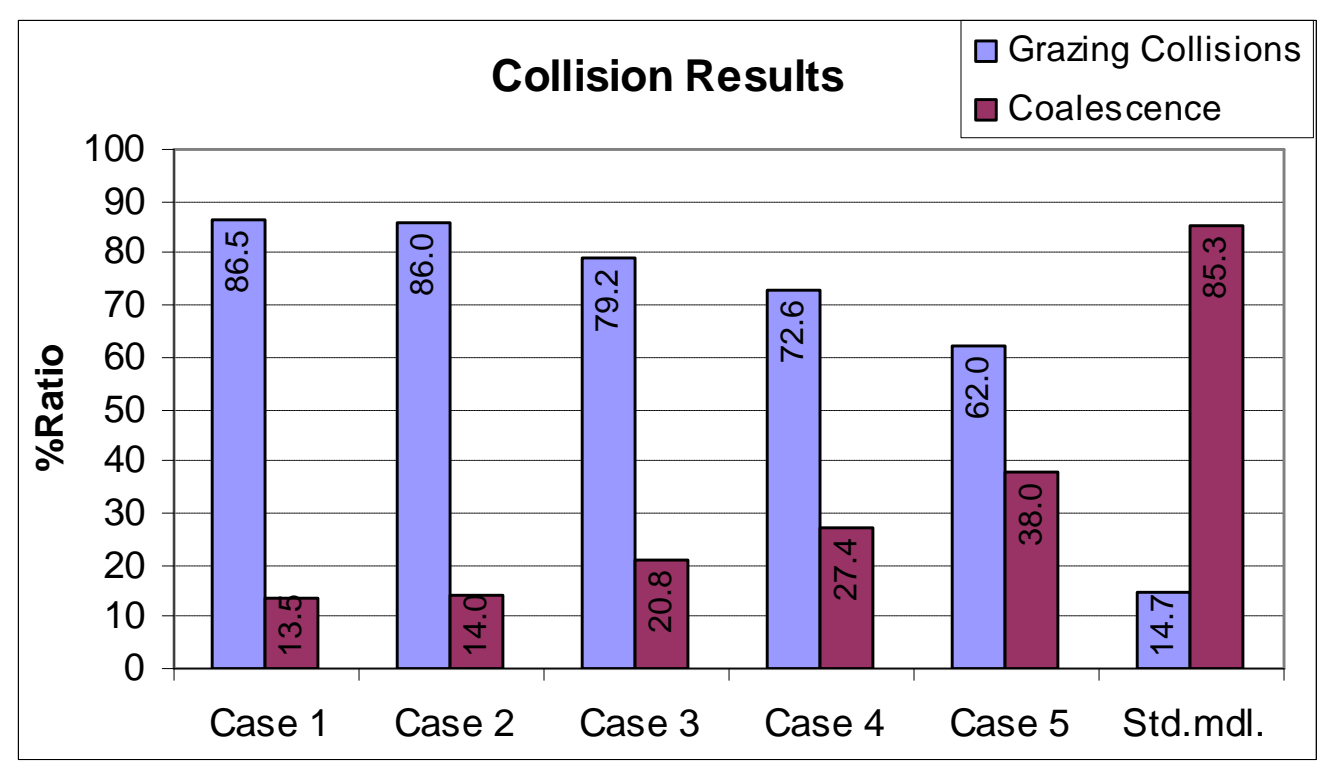

Figure 4 : Collision test results

The total parcel number has a dominant effect on collisions as shown in Fig.4. The total parcel number increased to 6000, 8000 and 10000 in Case 3, 4 and 5 respectively. For the Case 1 and 5 the computer times were 26 min and 81 minutes respectively. Though higher parcel numbers increase the computer time, it can be said that in numerical calculations, higher number of parcels would give more realistic results for collision of droplets. However limitation comes from computer capacity. The optimum parcel number for a representative simulation must be found before calculations. 
$\left[I^{1}\right.$
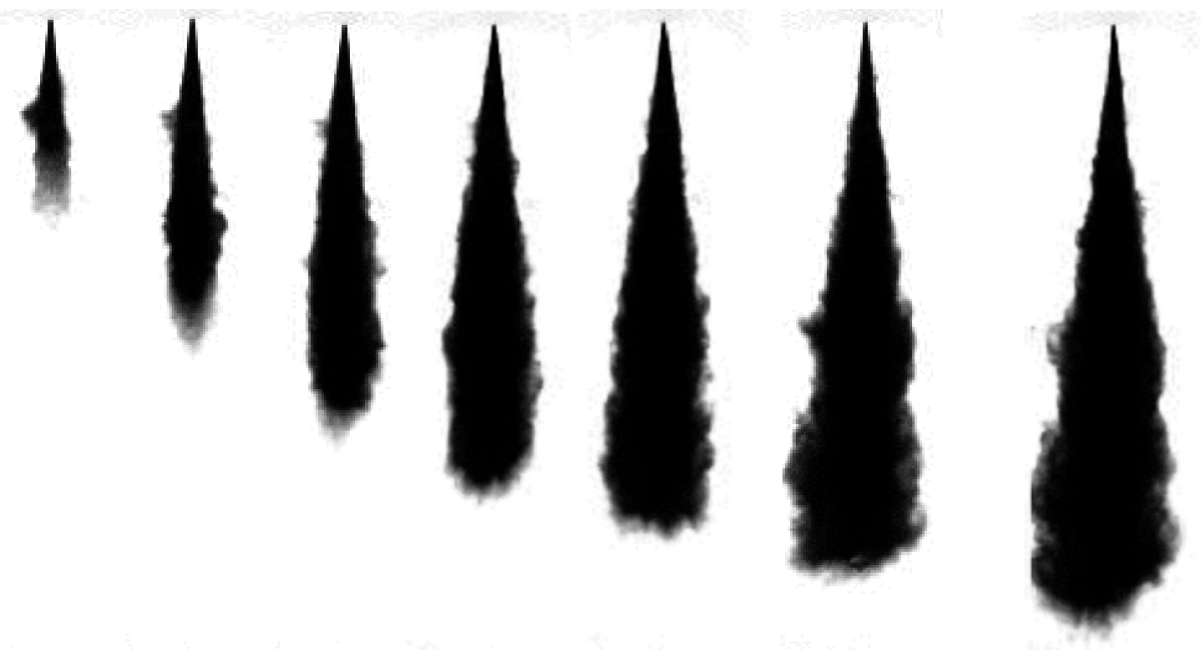

(a) Experimental results
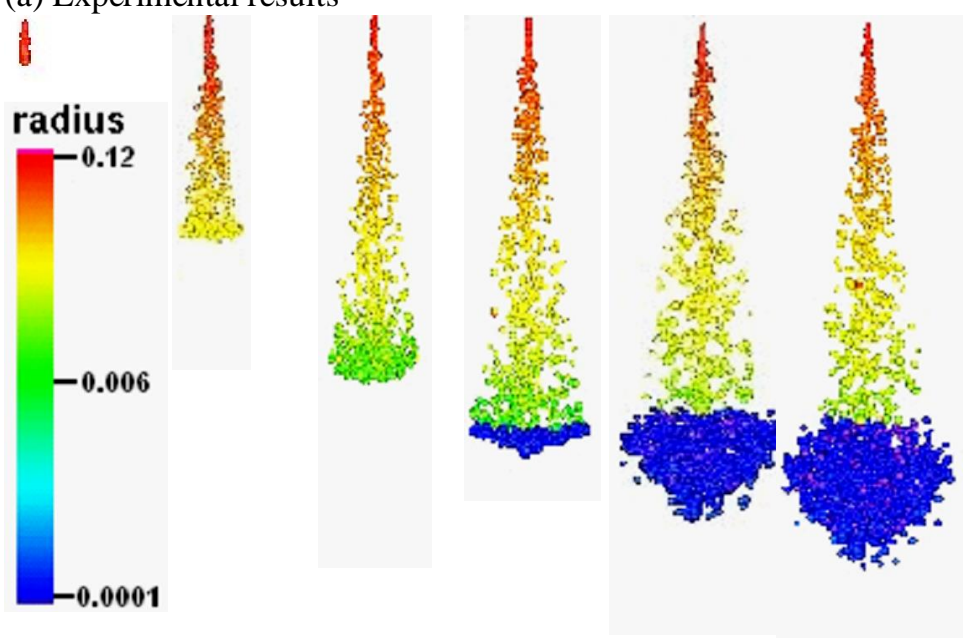

$0.063 \mathrm{~ms} \quad 0.125 \mathrm{~ms} \quad 0.188 \mathrm{~ms} \quad 0.250 \mathrm{~ms} \quad 0.313 \mathrm{~ms} \quad 0.375 \mathrm{~ms}$

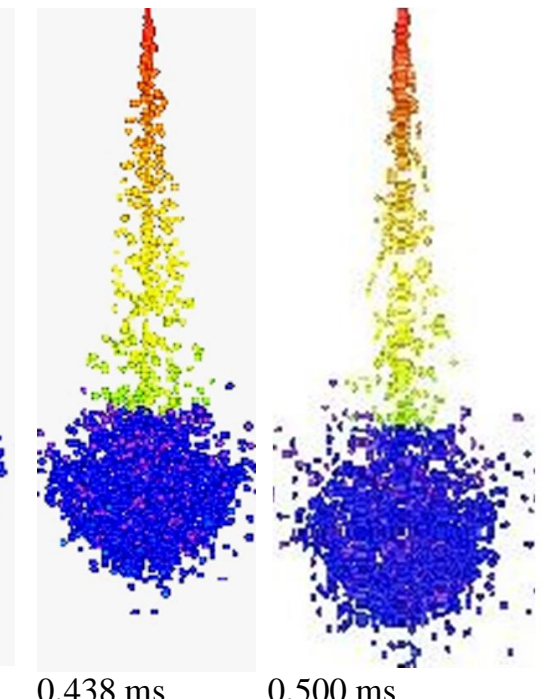

$0.438 \mathrm{~ms}$

$0.500 \mathrm{~ms}$

(b) Numerical results of KIVA3V R2 with new collision model

Figure 5: Spray development images of $9.5 \mathrm{mg}$ fuel injected to $3 \mathrm{MPa}$ and $300{ }^{\circ} \mathrm{K}$ constant volume vessel with $0.6 \mathrm{~ms}$ injection duration

To show numerical spray images of the KIVA model, the spray development obtained with numerical and experimental results are given in the Fig.5. KHRT breakup model, which produces new parcels as a result of breakup process and increase the total parcel number in the calculation, was used in numerical calculation. The code was started with 3000 parcel and it is automatically increased up to 7000 parcel in the calculation. The experimental results were obtained with a constant volume vessel of which details and experimental technique are presented in [10].

Taking into consideration spray penetration and development images, KIVA3V R2 numerical code results are in good agreement with experimental results. Since the droplet breakup is most dominant sub-model in spray dynamic, the droplet coalescence subsequently results with droplet breakup. Droplet collision modeling gets higher importance for intersecting sprays.

\section{Conclusions}

The advantage of the suggested model is that it derives impact parameter of the colliding droplets from the parcel velocity and position data. Though new model represents the physical process of collision more accurately, the results show that the model is dependent on parcel number used in the calculations. In the future, advances in computer technology may enable to use real droplet number instead of parcel representations for spray modeling.

The droplet collision model of the standard KIVA code needs to be further improved by reducing particle number dependence and introducing other droplet collision regimes such as bouncing, reflexive separation and shattering collision. The effect of break-up model dominates formation of spray and surpasses to see the effect of 
droplet collision. However, droplet collisions get important for intersecting sprays and it is needed to use a trajectory based model to obtain more accurate outcome of droplet collisions.

\section{References}

1. A.A. Amsden, P.J. OôRourke, ve T.D. Butler, KIVA-II: ñA Computer Program for Chemically Reacting Flows with Spraysò, Los Alamos National Laboratory Technical Report, (1989), LA-11560-MS.

2. Y. J. Jiang, A. Umemura, C. K. Law. ñAn Experimental Investigation on The Collision Behaviour of Hydrocarbon Dropletsò, Journal of Fluid Mechanics, (1992), Vol. 234, 171 İ̈ 190.

3. J. Qian, C.K. Law, ñRegimes of Coalescence and Separation in Droplet Collisionò, Journal of Fluid Mechanics, (1997), Vol. 331, 59-80.

4. P.J. OôRourke, ñCollective Drop Effects on Vaporizing Liquid Spraysò, PhD Thesis, (1981), Princeton University, USA.

5. N.Nordin, ñComplex Chemistry Modeling of Diesel Spray Combustionò, PhD Thesis, (2000), Chalmers University of Technology, Sweden.

6. D. P. Schmidt, C. J. Rutland, ñReducing Grid Dependency in Droplet Collision Modelingò, Journal of Engineering for Gas Turbines and Power, (2004), Vol. 126, 227-233

7. D. P. Schmidt, C. J. Rutland, ñA New Droplet Collision Algorithmò, Journal of Computational Physics, (2000), Vol. 164, 62ï 80.

8. A.Munnannur, ñDroplet Collision Modelling in Multi-Dimensional Engine Spray Computationsò, PhD Thesis, (2007), Universitiy of Wisconsin-Madison, USA.

9. A. Munnannur, R. D. Reitz, ñA New Predictive Model for Fragmenting and Non-fragmenting Binary Broplet Collisionsò, International Journal of Multiphase Flow, (2007), Vol. 33, 873 ï 896.

10. Ö.O. Takkēen and M.Ergeneman, ñExperimental Study on Diesel Spray Characteristics and Autoignition Processò, Journal of Combustion, Vol. 2011, Article ID, 528126. 